\title{
Optimal planting systems for cut gladiolus and stock production ${ }^{(1)}$
}

\author{
IFTIKHAR AHMAD(2)*, MUHAMMAD BEHZAD RAFIQ(2), AWAIS AHMAD(2), \\ MUHAMMAD QASIM(2), BILAL ABDULLAH ${ }^{(2)}$
}

\begin{abstract}
A study was conducted to elucidate the effect of different planting systems, videlicet (viz.) flat, ridge, and raised bed system on growth, yield and quality of gladiolus and stock. Corms of 'Rose Supreme' and 'White Prosperity' gladiolus and seedlings of 'Cheerful White', 'Lucinda Dark Rose Double' and 'Lucinda Dark Rose Single' stock were planted on different planting systems in individual experiments for each species. Gladiolus had similar good quality production irrespective of planting systems with numerical superiority of ridge planting, which produced longer stems with higher stem fresh weight, but delayed corm sprouting by ca. $1 \mathrm{~d}$ compared to raised bed or flat planting system. Among cultivars, 'Rose Supreme' produced higher number of florets per spike, taller stems with longer spikes, higher fresh weight of stems and higher number of cormels than 'White Prosperity'. Stock plants grown on flat beds produced stems with greater stem length, leaf area and fresh weight of stems compared to ridge or raised bed planting systems. Plants grown on ridges produced the highest stem diameter, number of leaves per plant, total leaf chlorophyll contents, and number of flowers per spike. 'Cheerful White' and 'Lucinda Dark Rose Double' performed best by producing good quality stems in shorter period compared to 'Lucinda Dark Rose Single'. In summary, gladiolus should be grown on ridges, while stock may be planted on flat beds for higher yields of better quality flowers.
\end{abstract}

Keywords: Gladiolus hybrids, Matthiola incana, flower quality, flat beds, ridge planting, yield.

\section{RESUMO}

SISTEMAS DE PLANTIO OTIMIZADOS PARA A PRODUÇÃO DE GLADÍOLOS DE CORTE E GOIVO

Estudo foi conduzido para elucidar o efeito de diferentes sistemas de plantação, sistema de canteiro plano, cume e elevado no crescimento, rendimento e qualidade de gladíolos e goivo. Cormos de gladíolo 'Rose Supreme' e 'Prosperidade branca' e as mudas de goivo 'Cheerful White', 'Lucindra Dark Rose Double' e 'Lucindra Dark Rose Single' foram plantadas em diferentes sistemas de plantio em experimentos individuais para cada espécie. O Gladíolo teve produção de boa qualidade, independentemente dos sistemas de plantação com superioridade numérica do plantio de cume, que produziram hastes mais longas com maior peso fresco do caule, mas atrasaram a brotação dos cormos por ca. de $1 \mathrm{~d}$, em comparação com canteiro levantado ou plantio plano. Entre as cultivares, «Rose Supreme» produziu maior número de floretes por espiga, hastes mais altas com espigas mais longas, maior peso fresco de hastes e maior número de cormos do que 'White Prosperity'. As plantas de goivo cultivadas em canteiros planos produziram hastes com maior comprimento de haste, área foliar e peso fresco de hastes em comparação com sistemas de plantação de cume ou canteiro levantado. As plantas cultivadas em cristas produziram o maior diâmetro do caule, número de folhas por planta, conteúdo total de clorofila foliar e número de flores por espiga. 〈Cheerful White〉 e 〈Lucindra Dark Rose Double〉 apresentaram o melhor desempenho, produzindo hastes de boa qualidade em um período mais curto em comparação com «Lucindra Dark Rose Singlè. Em resumo, o gladíolo deve ser cultivado em cumes, enquanto o goivo pode ser plantado em canteiros planos para maiores rendimentos de flores de melhor qualidade.

Palavras-chave: hibrídos de Gladiolus, Matthiola incana, qualidade floral, canteiros planos, ridge planting, produção.

\section{INTRODUCTION}

Gladiolus (Gladiolus L. hybrids), also known as 'Sword Lily' belongs to family Iridaceae, is an important bulbous specialty cut flower that is cultivated worldwide. It is commercially cultivated in different regions of Pakistan using different planting systems (SAFIULLAH and AHMED, 2001). Stock (Matthiola incana L.) belongs to family Brassicaceae and is being used as an annual specialty cut flower in different parts of the world (REGAN and DOLE, 2010) and recently been introduced in Pakistan for commercial production as specialty cut flower crop (RAFIQ, 2015). Introduction of new specialty cut flower crops provide more options to the growers and marketing stakeholders; however, growers are not well versed with production and postharvest protocols (DOLE et al., 2013). Various factors can improve the yield per unit area; however, production potential of any crop is linked with planting systems, so it is essential that optimum planting system be defined to maximize financial return and production (DOLE

DOI: http://dx.doi.org/10.14295/oh.v23i3.1107

${ }^{(1)}$ Received in 17/07/2017 and accepted in 19/09/2017

${ }^{(2)}$ University of Agriculture, Institute of Horticultural Sciences, Faisalabad, Pakistan. *Corresponding author: iahmad3@ncsu.edu; iftikharahmadhashmi@gmail.com

Licensed by CC BY 4.0 
and WILKINS, 2005). Planting systems strongly affect the yield of bulbous crops and help weed and disease control and mechanization (MARCINEK et al., 2013). Higher transpiration rate, reduced light penetration and low soil temperatures may also be prevalent at different planting systems (HATFIELD et al., 1998). Higher transpiration rates affect carbohydrate accumulation, which in turn could affect vase life of cut flowers (MOSADDEGHI et al., 2009).

For majority of bulbous crops, ridge planting system is widely used in different parts of the world due to good drainage and quickly warming up of soil during the day time to improve the temperature in plant root zone and to improve soil aeration (FAUSEY, 1990; REEDER, 1990; HATFIELD et al., 1998). However, ridge planting may increase field preparation costs and lower the area of production, therefore, flat planting is progressively adopted as means of reducing some of the negative effects of ridge planting (URI et al., 1999).

Different specialty cut flowers have specific planting systems to grow quality flower stems. For specialty cut flowers, very little work has been done so far in Pakistan or many other developing countries to find out the best suitable planting system to be grown under local conditions. Keeping in view the importance of these emerging specialty cut flower crops and availability of limited information regarding optimal planting system, the present study was conducted to compare different planting systems (flat, ridges and raised beds) to produce healthy plants with good quality flowering stems.

\section{MATERIALS AND METHODS}

\section{Plant material}

Corms of 'Rose Supreme' and 'White Prosperity' gladiolus were purchased from a well-reputed local importing firm, acclimatized at ambient temperature for a week on arrival, treated with $2 \mathrm{~g} \mathrm{~L}^{-1}$ fungicide solution for 10 min., dried under shade and sown either on flat beds, ridges, or raised beds next day without removing scales. Nursery of 'Cheerful White', 'Lucind a Dark Rose Double' and 'Lucinda Dark Rose Single' stock was raised in 128cell plastic plug trays containing silt, compost, and coco coir $(1: 1: 1 ; \mathrm{v} / \mathrm{v} / \mathrm{v})$ substrate. Seedlings of stock were either transplanted on flat beds, ridges, or raised beds at 2-4 true leaf stage.

\section{Experimental design and treatments}

Experiments were conducted on both species in split plot design having three replicates of 20 plants each. For gladiolus, plant to plant distance and depth of sowing was $5-6 \mathrm{~cm}$ in all experimental units in $60 \mathrm{~cm}$ spaced rows. Gladiolus cultivars were sown during $2^{\text {nd }}$ week of October. Seedlings of stock cultivars were transplanted in the field at 2-3 true leaf stage during $3^{\text {rd }}$ week of November after thorough soil preparation and addition of slow release compound fertilizer (250 kg ha-1, 20:20:20 NPK). Plant to plant and row to row distance was maintained at $30 \mathrm{~cm}$ for stock. Size of each plot was $3 \times 1.5 \mathrm{~m}$ for both species. All other cultural practices, viz. fertilization, irrigation, weeding, IPM, staking etc. were similar for all treatments during study period. Soil samples were randomly taken from both species experimental plots to evaluate physio-chemical properties ( $\mathrm{pH}, \mathrm{EC}$, organic matter and $\mathrm{N}, \mathrm{P}$ and $\mathrm{K}$ content) of the soil before addition of fertilizer. The study area climate was semi-arid, while soil type was a sandy loam, which had an organic matter content of $0.66 \%, \mathrm{pH}(8.78), \mathrm{EC}(2.50 \mathrm{dS}$ $\mathrm{m}^{-1}$ ), nitrogen (480 $\left.\mathrm{mg} \mathrm{kg}^{-1}\right)$, available phosphorus $(9.6 \mathrm{mg}$ $\mathrm{kg}^{-1}$ ) and exchangeable potassium (200 $\left.\mathrm{mg} \mathrm{kg}^{-1}\right)$.

\section{Observations}

Data were recorded for time to $50 \%$ sprouting (gladiolus only), production time (duration from sowing till ready to harvest), stem length, stem diameter, leaf area, sub-stomatal $\mathrm{CO}_{2}$ (stock only), leaf photosynthetic rate (stock only), leaf total chlorophyll contents (SPAD) (CCM-200 Plus chlorophyll meter), spike length, number of florets/flowers per spike, fresh and dry weight of a stem and vase life. Vase life was recorded in a postharvest evaluation laboratory maintained at $20 \pm 2{ }^{\circ} \mathrm{C}$ and $60 \pm 10 \%$ relative humidity under a photosynthetically active radiation flux of $\sim 15-20 \mu \mathrm{mol} \mathrm{m}{ }^{-2}$ $\mathrm{s}^{-1}$ from cool-white fluorescent lamps on a $12 \mathrm{~h}$ of photoperiod. For each species, stems were individually placed into glass vases containing $400 \mathrm{~mL}$ tap water. Stems were observed daily for visual appeal during the vase life evaluation period and were terminated when $50 \%$ or more florets/flowers on an inflorescence/stem exhibited unattractive appearance due to petal wilt, petal drying, or bent neck (AHMAD et al., 2011). Number, weight and diameter of cormels per clump were also recorded for gladiolus.

\section{Statistical analysis}

Both experiments were conducted in a split plot design with three replications. Data were analyzed using analysis of variance (ANOVA) procedures and General Linear Models procedures of SAS (version 9.3, SAS Inst., Inc., Cary, NC, USA). Means were compared using Fisher's least significant difference (LSD) at $p \leq 0.05$.

\section{RESULTS AND DISCUSSION}

\section{Gladiolus}

All planting systems used in this experiment produced uniform quality stems with similar growth, yield and quality characteristic of gladiolus. However, plants grown on ridges exhibited highest stem length for both tested cultivars (Table 1). However, ridge planting delayed sprouting by $1 \mathrm{~d}$ compared to other tested planting systems (data not presented). For other growth and yield characteristics, all planting systems produced similar results with numerical superiority of ridge planting, while 'Rose Supreme' had higher number of florets per spike, produced taller stems with longer spikes, and had higher fresh weight compared to 'White Prosperity' (Table 1). Moreover, 'Rose Supreme' produced three times higher number of cormels compared to 'White Prosperity'. No differences were found in sprouting percentage, spike diameter, vase life, and diameter or weight of cormels per clump (data not presented). 
Table 1. Effect of different planting systems on number of florets per spike, stem length, spike length, fresh and dry weight of a stem and number of cormels per clump of different cultivars of gladiolus. All data represent means of 15 plants.

\begin{tabular}{|c|c|c|c|c|c|c|c|}
\hline \multicolumn{2}{|c|}{ Treatments } & \multirow{2}{*}{$\begin{array}{l}\text { Number of } \\
\text { florets per } \\
\text { spike }\end{array}$} & \multirow{2}{*}{$\begin{array}{c}\text { Stem } \\
\text { length } \\
(\mathrm{cm})\end{array}$} & \multirow{2}{*}{$\begin{array}{l}\text { Spike } \\
\text { length } \\
(\mathrm{cm})\end{array}$} & \multirow{2}{*}{$\begin{array}{c}\text { Fresh weight } \\
\text { of a stem } \\
\text { (g) }\end{array}$} & \multirow{2}{*}{$\begin{array}{l}\text { Dry weight } \\
\text { of a stem } \\
\text { (g) }\end{array}$} & \multirow{2}{*}{$\begin{array}{l}\text { Number of } \\
\text { cormels per } \\
\text { clump }\end{array}$} \\
\hline Cultivars & $\begin{array}{l}\text { Planting } \\
\text { systems }\end{array}$ & & & & & & \\
\hline \multirow[t]{3}{*}{ 'Rose Supreme' } & Flat & $13.7 a b^{z}$ & $101.7 \mathrm{ab}$ & $37.6 \mathrm{a}$ & $80.5 \mathrm{ab}$ & $6.0 \mathrm{ab}$ & $36.8 \mathrm{a}$ \\
\hline & Ridges & $14.7 \mathrm{a}$ & $103.4 \mathrm{a}$ & $37.4 \mathrm{a}$ & $86.2 \mathrm{a}$ & $6.4 \mathrm{a}$ & $44.7 \mathrm{a}$ \\
\hline & Raised beds & $12.6 \mathrm{~b}$ & $99.7 \mathrm{ab}$ & $35.7 \mathrm{ab}$ & $67.5 \mathrm{~b}$ & $6.0 \mathrm{ab}$ & $41.0 \mathrm{a}$ \\
\hline \multirow[t]{3}{*}{ 'White Prosperity’ } & Flat & $12.7 \mathrm{~b}$ & $89.5 \mathrm{~cd}$ & $34.5 \mathrm{~b}$ & $71.6 \mathrm{~b}$ & $5.9 \mathrm{ab}$ & $17.3 \mathrm{~b}$ \\
\hline & Ridges & $12.1 \mathrm{~b}$ & $94.4 \mathrm{bc}$ & $33.6 \mathrm{~b}$ & $72.4 \mathrm{ab}$ & $5.7 \mathrm{~b}$ & $17.9 \mathrm{~b}$ \\
\hline & Raised beds & $9.8 \mathrm{c}$ & $99.7 \mathrm{ab}$ & $30.6 \mathrm{c}$ & $50.9 \mathrm{c}$ & $4.9 \mathrm{c}$ & $15.2 \mathrm{~b}$ \\
\hline \multicolumn{8}{|c|}{ Significance ${ }^{y}$} \\
\hline & Overall & $<0.0001$ & $<0.0001$ & $<0.0001$ & 0.0002 & 0.0019 & $<0.0001$ \\
\hline & Cultivar (C) & $<0.0001$ & $<0.0001$ & $<0.0001$ & 0.0025 & 0.0023 & $<0.0001$ \\
\hline & System (S) & NS & NS & NS & NS & NS & NS \\
\hline & $\mathrm{C} \times \mathrm{S}$ & 0.0002 & NS & 0.0274 & 0.0011 & 0.0103 & NS \\
\hline
\end{tabular}

${ }^{\mathrm{z}}$ Mean separation within columns by Fisher's LSD at $p \leq 0.05$.

${ }^{\text {y }} P$ values were obtained using General Linear Models (GLM) procedures of SAS (version 9.3; SAS Institute, Cary, NC) for significant effects of cultivars and planting systems.

NS Nonsignificant at $p>0.05$.

Results demonstrated that gladiolus can be successfully grown in any planting system used in this experiment in local agro-climatic and soil conditions of Faisalabad, Punjab, Pakistan. However, being taller and heavier crop while on flowering, it is advisable to grow using ridge planting system, which enables deep sowing of corms to avoid lodging and loss of quality (Personal observation). Moreover, it may improve soil aeration and aid in sprouting of corms particularly in heavy soils to produce more vigorous plants, which increased deposition of assimilates to the roots in comparison with other planting methods (MONNAF et al., 2010).

\section{Stock}

Stock plants grown on flat beds produced longer stems with greater leaf area, photosynthetic rate and fresh weight of stem (Tables 2 and 3). 
Table 2. Effect of different planting systems on production time, stem length, stem diameter, vase life, number of leaves per plant, leaf area and chlorophyll contents of different cultivars of stock. All data represent means of 15 plants.

\begin{tabular}{|c|c|c|c|c|c|c|c|c|}
\hline \multicolumn{2}{|c|}{ Treatments } & \multirow{2}{*}{$\begin{array}{l}\text { Production } \\
\text { time (days) }\end{array}$} & \multirow{2}{*}{$\begin{array}{c}\text { Stem } \\
\text { length }(\mathrm{cm})\end{array}$} & \multirow{2}{*}{$\begin{array}{c}\text { Stem } \\
\text { diameter } \\
(\mathbf{m m})\end{array}$} & \multirow{2}{*}{$\begin{array}{c}\text { Vase life } \\
\text { (days) }\end{array}$} & \multirow{2}{*}{$\begin{array}{l}\text { Number of } \\
\text { leaves } \\
\text { per plant }\end{array}$} & \multirow{2}{*}{$\begin{array}{l}\text { Leaf area } \\
\left(\mathrm{cm}^{2}\right)\end{array}$} & \multirow{2}{*}{$\begin{array}{l}\text { Chlorophyll } \\
\text { contents } \\
\left(\mathrm{mg} \mathrm{g}^{-1}\right)\end{array}$} \\
\hline Cultivars & $\begin{array}{l}\text { Planting } \\
\text { systems }\end{array}$ & & & & & & & \\
\hline \multirow{3}{*}{$\begin{array}{l}\text { 'Cheerful } \\
\text { White' }\end{array}$} & Flat & $128.5 \mathrm{~d}^{\mathrm{z}}$ & $51.8 \mathrm{a}$ & $7.3 \mathrm{cbd}$ & $9.9 \mathrm{a}$ & $30.5 \mathrm{~d}^{\mathrm{z}}$ & $46.4 \mathrm{a}$ & $121.9 \mathrm{a}$ \\
\hline & Ridges & $131.5 \mathrm{bc}$ & $48.9 \mathrm{~b}$ & $7.7 \mathrm{~b}$ & $9.7 \mathrm{a}$ & $28.9 \mathrm{de}$ & $43.6 \mathrm{ab}$ & $122.1 \mathrm{a}$ \\
\hline & $\begin{array}{l}\text { Raised } \\
\text { beds }\end{array}$ & $131.1 \mathrm{bcd}$ & $47.4 \mathrm{~b}$ & $7.5 \mathrm{cb}$ & $9.1 \mathrm{ab}$ & $27.1 \mathrm{e}$ & $41.0 \mathrm{~b}$ & $115.4 \mathrm{ab}$ \\
\hline \multirow{3}{*}{$\begin{array}{c}\text { 'Lucinda } \\
\text { Dark } \\
\text { Rose } \\
\text { Double' }\end{array}$} & Flat & $135.0 \mathrm{a}$ & $37.6 \mathrm{c}$ & $7.3 \mathrm{cbd}$ & $8.2 \mathrm{cde}$ & $34.9 \mathrm{bc}$ & $40.3 \mathrm{~b}$ & $59.1 \mathrm{~d}$ \\
\hline & Ridges & $133.3 \mathrm{a}$ & $35.0 \mathrm{~cd}$ & $9.2 \mathrm{a}$ & $8.8 \mathrm{bc}$ & $37.4 \mathrm{ab}$ & $34.0 \mathrm{c}$ & $68.3 \mathrm{~cd}$ \\
\hline & $\begin{array}{l}\text { Raised } \\
\text { beds }\end{array}$ & $132.1 \mathrm{~b}$ & $34.9 \mathrm{cde}$ & $7.7 \mathrm{~b}$ & $8.6 \mathrm{bcd}$ & $34.1 \mathrm{c}$ & $34.3 \mathrm{c}$ & $64.7 \mathrm{~d}$ \\
\hline \multirow{3}{*}{$\begin{array}{c}\text { 'Lucinda } \\
\text { Dark } \\
\text { Rose } \\
\text { Single' }\end{array}$} & Flat & $130.8 \mathrm{bcd}$ & $36.3 \mathrm{~cd}$ & $6.7 \mathrm{cbd}$ & 7.8 ed & $36.1 \mathrm{bc}$ & $18.2 \mathrm{~d}$ & $80.9 \mathrm{~cd}$ \\
\hline & Ridges & $129.3 \mathrm{~cd}$ & 34.5 de & $6.8 \mathrm{~cd}$ & $7.7 \mathrm{e}$ & $39.0 \mathrm{a}$ & $21.8 \mathrm{~d}$ & $92.3 \mathrm{bc}$ \\
\hline & $\begin{array}{l}\text { Raised } \\
\text { beds }\end{array}$ & $131.0 \mathrm{bcd}$ & $32.7 \mathrm{e}$ & $6.5 \mathrm{~d}$ & $7.5 \mathrm{e}$ & $36.7 \mathrm{abc}$ & $18.3 \mathrm{~d}$ & $77.5 \mathrm{~cd}$ \\
\hline \multicolumn{9}{|c|}{ Significance $^{\mathrm{y}}$} \\
\hline & Overall & 0.0003 & $<0.0001$ & $<0.0001$ & $<0.0001$ & $<0.0001$ & $<0.0001$ & $<0.0001$ \\
\hline & $\begin{array}{l}\text { Cultivar } \\
\text { (C) }\end{array}$ & 0.0002 & $<0.0001$ & $<0.0001$ & $<0.0001$ & $<0.0001$ & $<0.0001$ & $<0.0001$ \\
\hline & System (S) & NS & $<0.0001$ & 0.0430 & NS & 0.0281 & 0.0373 & NS \\
\hline & $\mathrm{C} \times \mathrm{S}$ & 0.0128 & NS & NS & NS & NS & NS & NS \\
\hline
\end{tabular}

${ }^{\mathrm{z}}$ Mean separation within columns by Fisher's LSD at $P \leq 0.05$.

${ }^{y} P$ values were obtained using General Linear Models (GLM) procedures of SAS (version 9.3; SAS Institute, Cary, NC) for significant effects of planting systems and cultivars.

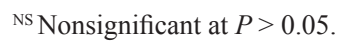

However, different cultivars behaved differently for different parameters regarding planting systems. 'Cheerful White' produced marketable stems earlier when grown in flat beds, while 'Lucinda Dark Rose
Double' produced early flowers when grown in raised beds. 'Lucinda Dark Rose Single' had no effect of different planting systems on production time. 
Table 3. Effect of different planting systems on number of flowers per spike, length of spike, flower diameter, substomatal $\mathrm{CO}_{2}$, photosynthetic rate, and fresh and dry weight of a stem od different cultivars of stock. All data represent means of 15 plants.

\begin{tabular}{|c|c|c|c|c|c|c|c|c|}
\hline \multicolumn{2}{|c|}{ Treatments } & \multirow[b]{2}{*}{$\begin{array}{c}\text { Number of } \\
\text { flowers per } \\
\text { spike }\end{array}$} & \multirow[b]{2}{*}{$\begin{array}{l}\text { Length of } \\
\text { spike } \\
\text { (cm) }\end{array}$} & \multirow[b]{2}{*}{$\begin{array}{c}\text { Flower } \\
\text { diameter } \\
(\mathbf{m m})\end{array}$} & \multirow[b]{2}{*}{$\begin{array}{l}\text { Sub-stomatal } \\
\qquad \mathrm{CO}_{2} \\
\left(\mu \mathrm{mol} \mathrm{m}{ }^{-2}\right. \\
\left.\mathrm{s}^{-1}\right)\end{array}$} & \multirow{2}{*}{$\begin{array}{c}\text { Photo- } \\
\text { synthetic } \\
\text { rate } \\
(\mu \mathrm{mol} \mathrm{O} \\
\left.\mathbf{m}^{-1} \mathbf{m i n}^{-1}\right)\end{array}$} & \multirow[b]{2}{*}{$\begin{array}{c}\text { Fresh } \\
\text { weight of a } \\
\text { stem } \\
\text { (g) }\end{array}$} & \multirow[b]{2}{*}{$\begin{array}{l}\text { Dry weight } \\
\text { of a stem } \\
\text { (g) }\end{array}$} \\
\hline Cultivars & $\begin{array}{l}\text { Planting } \\
\text { systems }\end{array}$ & & & & & & & \\
\hline \multirow{3}{*}{$\begin{array}{l}\text { 'Cheerful } \\
\text { White' }\end{array}$} & Flat & $13.3 \mathrm{~cd}^{\mathrm{z}}$ & $13.1 \mathrm{a}$ & $41.0 \mathrm{c}$ & $522.0 \mathrm{a}$ & $5.7 \mathrm{ab}$ & $6.0 \mathrm{a}$ & $13.3 \mathrm{~cd}$ \\
\hline & Ridges & $14.5 \mathrm{bc}$ & $13.0 \mathrm{a}$ & $48.3 \mathrm{a}$ & $571.7 \mathrm{a}$ & $5.5 \mathrm{ab}$ & $5.7 \mathrm{ab}$ & $14.5 \mathrm{bc}$ \\
\hline & $\begin{array}{l}\text { Raised } \\
\text { beds }\end{array}$ & $13.5 \mathrm{~cd}$ & $13.5 \mathrm{a}$ & $44.8 \mathrm{~b}$ & $417.0 \mathrm{~b}$ & $3.4 \mathrm{abc}$ & $4.3 \mathrm{c}$ & $13.5 \mathrm{~cd}$ \\
\hline \multirow{3}{*}{$\begin{array}{l}\text { 'Lucinda } \\
\text { Dark } \\
\text { Rose } \\
\text { Double' }\end{array}$} & Flat & $13.9 \mathrm{c}$ & $10.5 \mathrm{~b}$ & $42.0 \mathrm{cb}$ & $419.7 \mathrm{~b}$ & $6.4 \mathrm{a}$ & $5.3 \mathrm{abc}$ & $13.9 \mathrm{c}$ \\
\hline & Ridges & $18.0 \mathrm{a}$ & $11.1 \mathrm{~b}$ & $40.7 \mathrm{c}$ & $404.0 \mathrm{~b}$ & $3.8 \mathrm{abc}$ & $4.8 \mathrm{bc}$ & $18.0 \mathrm{a}$ \\
\hline & $\begin{array}{l}\text { Raised } \\
\text { beds }\end{array}$ & $15.9 \mathrm{~b}$ & $10.6 \mathrm{~b}$ & $41.1 \mathrm{c}$ & $392.3 \mathrm{~b}$ & $2.2 \mathrm{c}$ & $4.2 \mathrm{c}$ & $15.9 \mathrm{~b}$ \\
\hline \multirow{3}{*}{$\begin{array}{l}\text { 'Lucinda } \\
\text { Dark } \\
\text { Rose } \\
\text { Single' }\end{array}$} & Flat & $11.8 \mathrm{~d}$ & $11.0 \mathrm{~b}$ & $34.4 \mathrm{~d}$ & $421.3 \mathrm{~b}$ & $6.5 \mathrm{a}$ & $4.4 \mathrm{c}$ & $11.8 \mathrm{~d}$ \\
\hline & Ridges & $13.2 \mathrm{~cd}$ & $10.0 \mathrm{bc}$ & $32.0 \mathrm{~d}$ & $410.3 \mathrm{~b}$ & $2.6 \mathrm{bc}$ & $4.3 \mathrm{c}$ & $13.2 \mathrm{~cd}$ \\
\hline & $\begin{array}{l}\text { Raised } \\
\text { beds }\end{array}$ & $12.9 \mathrm{~cd}$ & $8.4 \mathrm{c}$ & $31.7 \mathrm{~d}$ & $388.7 \mathrm{~b}$ & $2.9 \mathrm{bc}$ & $2.9 \mathrm{~d}$ & $12.9 \mathrm{~cd}$ \\
\hline \multicolumn{9}{|c|}{ Significance $^{y}$} \\
\hline & Overall & $<0.0001$ & $<0.0001$ & $<0.0001$ & 0.0030 & 0.0470 & $<0.0001$ & $<0.0001$ \\
\hline & $\begin{array}{l}\text { Cultivar } \\
\text { (C) }\end{array}$ & $<0.0001$ & $<0.0001$ & $<0.0001$ & 0.0007 & NS & $<0.0001$ & 0.0001 \\
\hline & System (S) & 0.0330 & NS & NS & 0.0343 & 0.0036 & 0.0087 & $<0.0001$ \\
\hline & $\mathrm{C} \times \mathrm{S}$ & 0.0140 & NS & 0.0008 & NS & NS & NS & NS \\
\hline
\end{tabular}

Stock plants grown on ridges had greater stem diameter for all tested cultivars, but had similar vase life irrespective of the planting systems. Mahmood et al. (2012) found that black cumin (Nigella sativa L.) produced taller plants when grown on flat beds, which might be due to competition for light and nutrients. Moreover, in roses, two parallel rows planting produced better results compared to three or four rows (MÉNARD and DANSEREAU, 1992). Similarly, fennel (Foeniculam vulgare L.) also produced taller stems, when grown on flat beds (YADAV and KHURANA, 1999; AYUB et al., 2008).

Leaf area of 'Cheerful White' and 'Lucinda Dark Rose Double' was significantly increased when grown on flat planting system (46.4 and $40.3 \mathrm{~mm}^{2}$, respectively; Table 2). Stock plants grown on ridges had higher number of leaves, leaf total chlorophyll contents, number of flowers per spike, spike length and dry weight of stem compared to flat or raised beds. For flower diameter, 'Cheerful White' had higher diameter when grown in flat planting, while both 'Lucinda Dark Rose' cultivars had greater flower diameter when grown on ridges (Table 3). Several researchers have reported that growth vigor like leaf area and dry weight are significantly affected when different planting methods were used (URI et al., 1999; PAZOKI et al., 2013). Tuncturk et al. (2005) and Monnaf et al. (2010) reported more number of flowers per spike when plants were grown on ridges, probably due to reduced lodging and better agricultural practices (MAHMOOD et al., 2012). 'Cheerful White' and 'Lucinda Dark Rose Double' stock produced better quality flowers compared to 'Lucinda Dark Rose Single'. 


\section{CONCLUSIONS}

Gladiolus should preferably be grown on ridges, while stock may be planted on flat or ridge planting system for higher yield and good quality cut stems in fields. 'Rose Supreme' gladiolus and 'Cheerful White' and 'Lucinda Dark Rose Double' performed best irrespective of planting system.

\section{ACKNOWLEDGEMENTS}

Authors gratefully acknowledge Prof. Dr. John M. Dole, Department Head, Horticultural Science, North Carolina State University, Raleigh, NC, USA, for provision of stock cultivars seeds for the study.

\section{AUTHORS CONTRIBUTIONS}

IA: Planned the research experiments, and wrote the manuscript. MBR: Performed the experiment on stock cultivars and collected and analyzed data. AA: Performed the experiment on gladiolus cultivars and collected and analyzed data. MQ: Guided in experiment planning and reviewed the manuscript. BA: Reviewed the manuscript.

\section{REFERENCES}

AHMAD, I.; JOYCE, D.C.; FARAGHER, J.D. Physical stem-end treatment effects on cut rose and acacia vase life and water relations. Postharvest Biology and Technology, v.59, p.258-264, 2011.

AYUB, M.; NADEEM, M.A.; TANVEER, A.; TAHIR, M.; SAQIB, M.T.Y.; NAWAZ. R. Effect of different sowing methods and times on the growth and yield of fennel (Foeniculam vulgare). Pakistan Journal of Botany, v.40, p.259-264, 2008.

DOLE, J.M.; WILKINS, H.F. Floriculture: Principles and Species, $2^{\text {nd }}$ ed. Prentice Hall, Upper Saddle River, NJ, USA, 2005.

DOLE, J.M.; CARLSON, A.S.; CRAWFORD, B.D.; McCall, I.F. Vase life of new cut flowers. Acta Horticulturae, v.1000, p.63-70, 2013.

FAUSEY, N.R. Experience with ridge-till on slowly permeable soils in Ohio. Soil \& Tillage Research, v.18, p.195205,1990 .

HATFIELD, J.L.; ALLMARAS, R.R., REHM, G.W.; LOWERY, B. Ridge tillage for corn and soybean production: environmental quality impacts. Soil \& Tillage Research, v.48, p.145-154, 1998.

MAHMOOD, T.; IDRESS, M.; ASLAM, M.; REHMAN, H.S.; AKRAM, H.M.; SATTAR, A.; ABBAS, S.; FERDOSI, M.F.H. Growth and yield attributes of black cumin $(\mathrm{Ni}-$ gellla sativa L.) as affected by sowing dates and methods. Mycopathology, v.10, p.83-86, 2012.
MARCINEK, B.; HETMAN, J.; KOZAK, D. Influence of cultivation method and bulbs planting depth on the growth and yielding of tulips. Acta Scientiarum Polonorum Horticulture, v.12, p.97-110, 2013.

MÉNARD, C.; DANSEREAU, B. Influence of photoshynthetic photon flux density and planting scheme on growth and development of cultivar 'Royalty' roses. Scientia Horticulturae, v. 50, p. 197-207, 1992.

MONNAF, M.A.; RAHIM, M.A.; HOSSAIN, M.M.A.; ALAM, M.S. Effect of planting method and rhizome size on the growth and yield of ginger. Journal of Agroforestry and Environment, v.4, p.73-76, 2010.

MOSADDEGHI, M.R.; MAHBOUBI, A.A.; SAFADOUST, A. Short-term effects of tillage and manure on some soil physical properties and maize root growth in a sandy loam soil in western Iran. Soil and Tillage Research, v.104, p.173-179, 2009.

PAZOKI, A.; KARIMINEJAD, M.; TARGHI, A.F. Effect of planting patterns on yield and some agronomical traits in saffron (Crocus sativus L.) under different irrigation intervals in Shahr-e-Rey Region. International Journal of Farming and Allied Sciences, v.2, p.13631368, 2013.

RAFIQ, M.B. Pre and postharvest evaluation of various native and exotic ornamentals for specialty cut flower production. M.Sc. (Hons.) Thesis, Institute of Horticultural Sciences, University of Agriculture, Faisalabad, Pakistan, 2015.

REEDER, R.C. Extension programs and farmer experiences with ridge tillage. Soil \& Tillage Research, v.18, p.283-293, 1990.

REGAN, E.M.; DOLE, J.M. Postharvest handling procedures of Matthiola incana 'Vivas Blue'. Postharvest Biology and Technology, v.58, p.268-273, 2010.

SAFIULLAH; AHMED, M.J. Evaluation of exotic cultivars of gladiolus (Gladiolus grandiflorus) under Rawalakot conditions. Sarhad Journal of Agriculture, v.17, p.172174, 2001.

TUNCTURK, M.; EKIN, Z.; TURKOZU, D. Response of black cumin (Nigella sativa L.) to different seed rates on growth, yield components and essential oil content. Journal of Agronomy, v.4, p.216-219, 2005.

URI, N.D.; ATWOOD, J.D.; SANABRIA, J. The environmental benefits and costs of conservation tillage. Environmental Geology, v.38, p.111-125, 1999.

YADAV, B.D.; KHURANA, S.C. Effect of planting methods and sowing dates on growth and yield of fennel. Haryana Agricultural University Journal of Research, v.29, p.81-87, 1999. 